\title{
SPR Chemosensors Based on D-Shaped POFs and MIPs: Investigation on Optimal Thickness of the Buffer Layer $^{\dagger}$
}

\author{
Nunzio Cennamo ${ }^{1, *}$, Letizia De Maria ${ }^{2}$, Maria Pesavento ${ }^{3}$, Francesco Mattiello ${ }^{1}$, \\ Cristina Chemelli ${ }^{2}$ and Luigi Zeni ${ }^{1, *}$ \\ 1 Department of Industrial and Information Engineering, University of Campania Luigi Vanvitelli, \\ 81031 Aversa, Italy; francesco.mattiello@unicampanai.it \\ 2 RSE spa, Via Raffaele Rubattino, 20134 Milano, Italy; Letizia.DeMaria@rse-web.it (L.D.M.); \\ Cristina.Chemelli@rse-web.it (C.C.) \\ 3 Department of Chemistry, University of Pavia, Via Taramelli 12, 27100 Pavia, Italy; \\ maria.pesavento@unipv.it \\ * Correspondence: nunzio.cennamo@unicampania.it (N.C.); luigi.zeni@unicampania.it (L.Z.) \\ + Presented at the 5th International Symposium on Sensor Science (I3S 2017), Barcelona, Spain, \\ 27-29 September 2017.
}

Published: 13 December 2017

A comparative analysis of different optical sensing platforms, designed for chemical applications and based on molecularly imprinted polymers (MIPs), is presented. The optical platforms are based on surface plasmon resonance (SPR) in a D-shaped plastic optical fiber (POF), with a buffer layer between the exposed POF core and the thin gold film (60 nm thick). These optical platforms are realized by removing the cladding of POFs along half circumference, spin coating a buffer layer on the exposed core (with different velocities to obtain different thicknesses), and finally sputtering a thin gold film. The buffer layer proposed in this analysis is the photoresist Microposit S1813, with a refractive index greater than the one of the POF core. This photoresist buffer layer is required in order to increase the performances of the sensor.

We intend to show how, in the refractive index range used for chemical applications based on MIPs, the sensor's performances change when the thickness of the photoresist layer changes. The results show that when the thickness of the photoresist layer decreases, the sensor's sensitivity increases. The experimental results are congruent with the numerical studies and this analysis is instrumental for chemical applications.

Conflicts of Interest: The authors declare no conflict of interest.

(C) 2017 by the authors. Licensee MDPI, Basel, Switzerland. This article is an open access article distributed under the terms and conditions of the Creative Commons Attribution (CC BY) license (http://creativecommons.org/licenses/by/4.0/). 